\title{
Wag the dog: Skepticism on seizure alert canines
}

Michael J. Doherty, MD; and Alan M. Haltiner, PhD, American Academy of Neurology 2007; 68: 309.

Some dogs can signal human seizure activity. Seizure response dogs (SRDs) recognize and respond to ongoing seizure and postseizure compromise, whereas seizure alert dogs (SADs) may warn of impending seizure. I-5 Canine companions may have an additional stress reduction benefit that can lead to decreased seizure frequency. 5 In this report we describe a dog that warns of psychogenic nonepileptiform events (PNES) and critically examine the SAD literature.

Case study. According to the couple that owns her, their 2-year-old Blue Heeler can both alert and respond to seizure activity. The owners (who gave permission to use and publish these data) report that she lies upon the wife, across her chest, both prior to and during her convulsive seizures. Her husband, who also has seizures that the dog can predict, says the dog fetches help from neighbors after seizures or in times of crisis.

The wife underwent video EEG telemetry (VEEG). Her recorded events included anterior-to-posterior plane pelvic bucking, head-bobbling movements, psychomotor unresponsiveness, and postevent flaccidity. Her Minnesota Multiphasic Personality Inventory was filled backward and as such was uninterpretable. The diagnostic consensus at our multidisciplinary epilepsy conference was that her events were PNES. The dog was not present during the telemetry, and although the husband seized during his wife's stay, his event was not captured on video.

Discussion. This Blue Heeler is perhaps the third with an ability to predict PNES.2,4 Unfortunately, SAD studies have never objectively shown that dogs can predict seizures and fail to address data contamination with patients with PNES.2,4 The largest SAD survey, self-reported via parental survey of children with epilepsy, studied a population in which patients with PNES are less likely. From this study, $20 \%(n=9)$ of the family dogs warned of impending seizures without any prior training. The authors state the dogs' "anticipatory behaviors were never demonstrated without a subsequent seizure" (i.e., specificity was $100 \%$ ), whereas sensitivity was $80 \%{ }^{3}$ These outstanding figures suggest a need for retesting using different study designs and demographics.

In a different study of 63 adults with epilepsy, 29 had dogs, and of those dogs, 31\% responded to seizures and $10 \%$ (n = 3) alerted prior to seizures. 5 The authors conclude that although their numbers were too small to study statistically, further study, with dogs accompanying their owners during VEEG, was warranted, so dog responses might be better studied.

In a different study and the only one in which dog responses were studied objectively and independently, two dogs were observed during their owner's VEEG stays: The first failed to warn of impending events for seven of eight seizures; the second alerted the patient prior to a documented psychogenic event. ${ }^{4}$ These objective data are different from the $100 \%$ specific and $80 \%$ sensitive abilities of the SAD discussed in the pediatric survey. ${ }^{3}$ The authors cautiously conclude that the EEG telemetry unit may not be the ideal place for SADs to warn of events. An alternative explanation is that dogs are not prescient, and the really interesting data may not be what the dogs do but what the humans did.

Apart from the two patients and their dogs monitored in the VEEG unit, the entire literature is self-reported via diaries or surveys. ${ }^{2-5}$ Self-reported and even performance-based neuropsychological profiles measured in patients with PNES often do not correlate with independent objective measurements. ${ }^{6}$ This concern could contaminate much of the self-reported literature on SAD. Perhaps the question to answer is what the dogs respond to, as assessed by double-blinded observers watching dog and human behaviors during VEEG telemetry. 
Lots of questions are raised: If dogs can predict PNES, could events be viewed as conditioned responses to stereotyped dog behaviors? If teddy bears present during adult VEEG telemetry correlate with PNES, might SADs similarly prove red flags for potential patients with PNES?7 Do the dogs perceive a stimulus, perhaps an audible frequency or a peculiar odor, that represents seizure (or pseudoseizure) onset? Are dogs detecting early ictus, and are the families or patients under the impression this is preseizure? Given the costs of training SAD dogs, should patients inquiring about SADs be specifically screened with VEEG for PNES? Do SADs change the frequency of PNES?

A dog's companionship can be reassuring and relaxing and may cut down on seizure frequency. The ability for a dog to obtain help during or after a seizure could prove lifesaving. Without objectively clarifying if patients have epilepsy or PNES, the current literature fails to support that canines can warn of impending seizures.

\section{References}

I. EpilepsyUSA staff. Partnership makes service dogs available for people with epilepsy. Epilespy Foundation (online). Available at: www.epilepsyfoundation.org/epilepsyusa/ucbdogs200604.cfm; accessed July 6, 2006.

2. Strong V, Brown SW, Walker R. Seizure-alert dogs: fact or fiction? Seizure 1999;8:62-65.

3. Kirton A, Wirrell E, Zhang J, Hamikawa L. Seizure-alerting and -response behaviors in dogs living with epileptic children. Neurology 2004;62:2303-2305.

4. Ortiz R, Liporace J. "Seizure-alert dogs": observations from an inpatient video/EEG unit. Epilepsy Behav 2005;6:620-622.

5. Dalziel DJ, Uthman BM, McGorray SP, Reep RL. Seizure-alert dogs: a review and preliminary study. Seizure 2003;12:II5-120.

6. Binder LM, Kindermann SS, Heaton RK, Salinsky MC. Neuropsychologic impairment in patients with nonepileptic seizures. Arch Clin Neuropsychol 1998; 13:513-522.

7. Burneo JG, Martin R, Powell T, et al. Teddy bears: an observational finding in patients with non-epileptic events. Neurology 2003;61:714-7I5.

From the Swedish Epilepsy Center, 80I Broadway, Suite 90I, Seattle, WA 98I22

Copyright (C 2007 by AAN Enterprises, Inc. 